\title{
Simultaneous determination of perfluoroalkyl substances and bile acids in human serum using ultra-high-performance liquid chromatography-tandem mass spectrometry
}

\author{
Samira Salihović ${ }^{1,2} \cdot$ Alex M. Dickens $^{3} \cdot$ Ida Schoultz $^{1} \cdot$ Frida Fart $^{1} \cdot$ Lisanna Sinisalu $^{2} \cdot$ Tuomas Lindeman $^{3}$. \\ Jonas Halfvarson ${ }^{4}$ Matej Orešič ${ }^{1,3} \cdot$ Tuulia Hyötyläinen $^{2}$
}

Received: 7 September 2019 /Revised: 22 October 2019 / Accepted: 6 November 2019 / Published online: 23 November 2019

(C) The Author(s) 2019

\begin{abstract}
There is evidence of a positive association between per- and polyfluoroalkyl substances (PFASs) and cholesterol levels in human plasma, which may be due to common reabsorption of PFASs and bile acids (BAs) in the gut. Here we report development and validation of a method that allows simultaneous, quantitative determination of PFASs and BAs in plasma, using $150 \mu \mathrm{L}$ or $20 \mu \mathrm{L}$ of sample. The method involves protein precipitation using 96-well plates. The instrumental analysis was performed with ultraperformance liquid chromatography-tandem mass spectrometry (UHPLC-MS), using reverse-phase chromatography, with the ion source operated in negative electrospray mode. The mass spectrometry analysis was carried out using multiple reaction monitoring mode. The method proved to be sensitive, robust, and with sufficient linear range to allow reliable determination of both PFASs and BAs. The method detection limits were between 0.01 and $0.06 \mathrm{ng} \mathrm{mL}^{-1}$ for PFASs and between 0.002 and $0.152 \mathrm{ng} \mathrm{mL}^{-1}$ for BAs, with the exception of glycochenodeoxycholic acid $\left(0.56 \mathrm{ng} \mathrm{mL}^{-1}\right)$. The PFAS measured showed excellent agreement with certified plasma PFAS concentrations in NIST SRM 1957 reference serum. The method was tested on serum samples from 20 healthy individuals. In this proof-of-concept study, we identified significant associations between plasma PFAS and BA levels, which suggests that PFAS may alter the synthesis and/or uptake of BAs.
\end{abstract}

Keywords Bile acids $\cdot$ Human serum $\cdot$ LC $\cdot$ MS $\cdot$ Perfluoroalkyl substances $\cdot$ PFAS

\section{Introduction}

Published in the topical collection Current Progress in Lipidomics with guest editors Michal Holčapek, Gerhard Liebisch, and Kim Ekroos.

Electronic supplementary material The online version of this article (https://doi.org/10.1007/s00216-019-02263-6) contains supplementary material, which is available to authorized users.

Tuulia Hyötyläinen

tuulia.hyotylainen@oru.se

1 School of Medical Sciences, Örebro University, 702 81 Örebro, Sweden

2 School of Science and Technology, Örebro University, 702 81 Örebro, Sweden

3 Turku Bioscience Centre, University of Turku and Åbo Akademi University, 20520 Turku, Finland

4 Department of Gastroenterology, Faculty of Medicine and Health, Örebro University, 70281 Örebro, Sweden
In clinical studies, both in metabolomics and in environmental exposure studies, the volume of sample available for analysis is often restricted. Thus, it is desirable that the analytical methods can assay multiple substance classes simultaneously, while using as small volume of sample as possible. This presents a challenge, both regarding the chemical characteristics of the compounds that can be quantitatively covered by a single extraction and/or analytical method, as well as in terms of the concentration range of said substances which can be assayed for. While several methods have been developed for untargeted analyses which can simultaneously assay a large number of metabolites, it remains problematic to analyze simultaneously both specific metabolites and exogenous compounds (such as environmental pollutants), with the main challenge typically being the low concentrations of the latter.

In this study, we demonstrate the development of a targeted method for the analysis of two distinct compound classes, 
namely (i) endogenous bile acids (BAs) and (ii) exogenous per- and polyfluoroalkyl substances (PFASs) in human serum or plasma. BAs are metabolites that facilitate the digestion and absorption of lipids in the small intestine and they are also important metabolic regulators involved in the maintenance of lipid and glucose homeostasis [1,2]. PFASs, on the other hand, are a group of man-made chemicals that have been widely used since the 1950s in both household and industrial products. PFASs also have long biological half-lives, and are readily detected in humans [3-7]. Structurally, several PFAS compounds resemble endogenous fatty acids, with fluorine substitution in place of hydrogen. Biologically, the two compound groups share some common features. It is well known that BAs that are excreted into the intestine are reabsorbed, and similar enterohepatic circulation has been suggested for PFASs $[8,9]$.

Recently, the European Food Safety Authority (EFSA) reported that the positive association of perfluorooctane sulfonate (PFOS) and perfluorooctanoic acid (PFOA), the two most common PFASs, with total cholesterol serum levels, as observed in several studies, may result from a possible common reabsorption of bile acids, PFOS, and PFOA from the gut and shared membrane transport pathways into the liver (https:// www.efsa.europa.eu/sites/default/files/news/efsa-contam3503.pdf). Interestingly, it has been hypothesized that 7-alphahydroxylase (CYP7A1), which catalyzes the first and ratelimiting step in the formation of BAs from cholesterol, may be downregulated by PFASs $[9,10]$. This may lead to increased re-uptake of BAs, which would generate negative feedback loops via the farnesyl-X-receptor and subsequently reduce their de novo synthesis. Indeed, PFHxS and PFOS have been shown to decrease fecal BA excretion [11]. Thus, current knowledge already suggests concomitant reabsorption of BAs and PFASs in the intestine and that this could play a role in the observed association between serum levels of PFAS and cholesterol. At present, there is, however, limited empirical data demonstrating such a relationship.

Several methods have been developed for the quantitative determination of BAs and PFASs, using separate analytical methods [12-14]. Most of the methods include sample preparation using either (i) protein precipitation, often combined with further clean-up steps (e.g., phospholipid removal), particularly for PFASs, (ii) liquid-liquid extraction, or (iii) solidphase extraction. Such analyses are performed predominantly with various LC-MS/MS methods, typically using reversedphase LC in combination with triple quadrupole MS in multiple reaction monitoring mode. For PFAS analyses, the sample volumes required for analysis are typically several hundreds of microliters, while for BAs, smaller volumes are typically sufficient.

In this study, the aim was to develop and validate a quantitative method covering both BAs and PFASs in a single analysis, minimizing the required volume of serum. The method was validated by analysis of serum samples from healthy subjects, where the associations between PFAS and BA levels were investigated.

\section{Experimental}

\section{Chemicals}

Ammonium acetate $\left(\mathrm{NH}_{4} \mathrm{Ac}\right)$ was obtained from SigmaAldrich (St. Louis, USA). Methanol (MeOH) and acetonitrile (ACN) (both HPLC grade with purity greater than 99\%) were obtained from Fisher Scientific UK (Loughborough, UK). Acetonitrile (Optima® LC-MS grade) and formic acid (98$100 \%$ ) were purchased from Sigma-Aldrich (Steinheim, Germany). The MilliQ water used to make the mobile phase was $18.2 \mathrm{M} \Omega$. LC vials and OstroTM 96-well plate $(25 \mathrm{mg}$ $1 / \mathrm{pkg}$ ) were purchased from Waters (Waters Corporation, Milford, USA). Newborn bovine serum (New Zealand) was purchased from Sigma-Aldrich and stored frozen $\left(\leq-20^{\circ} \mathrm{C}\right)$ until analysis. For quality assurance (QA), standard reference material serum SRM 1957 was purchased from the National Institute of Standards and Technology (NIST) at the US Department of Commerce (Washington, DC, USA). The SRM sample was stored frozen $\left(\leq-20^{\circ} \mathrm{C}\right)$ until analysis. The quality control (QC) reference sample consisted of pooled human plasma collected from blood donors at Örebro University Hospital (Örebro, Sweden), and stored frozen ( $\leq$ $-20{ }^{\circ} \mathrm{C}$ ) until analysis.

Abbreviations of target analytes are presented in Table 1. ${ }^{13} \mathrm{C}$-labeled PFAS internal standards (IS), ${ }^{13} \mathrm{C}$-labeled performance standards, and native calibration standards (perfluorocarboxylic acids (PFCAs) and perflurosulfonic acids (PFSAs)) were purchased from Wellington Laboratories (Guelph, Ontario, Canada). CA, CDCA, DCA, DHCA, GCA, GCDCA, LCA, TCA, TCDCA, TDCA, TDHCA, THCA, THDCA, TLCA, and TUDCA were obtained from Sigma-Aldrich (St. Louis, MO, USA); HDCA, HCA, $\alpha$ MCA, $\beta$ MCA, $\omega \mathrm{MCA}, 7-$ oxo-HDCA, 7oxo-DCA, 12-oxo-LCA, T $\alpha$ MCA, T $\beta$ MCA, T $\omega \mathrm{MCA}$, GDHCA, GHCA, and GHDCA from Steraloids (Newport, RI, USA); GLCA and GUDCA from Calbiochem (Gibbstown, NJ, USA); and GDCA and UDGA from Fluka (Buchs, Switzerland). Internal standards CA-d4, LCA-d4, UDCA-d4, CDCA-d4, DCA-d4, GCA-d4, GLCA-d4, GUDCA-d4, and GCDCA-d4 were obtained from Qmx Laboratories Ltd. (Essex, UK). All standards were prepared in methanol and stored refrigerated $\left(4^{\circ} \mathrm{C}\right)$.

\section{Samples}

The serum samples $(n=20)$ were from blood donors at $\geq 55$ years of age, without any gastrointestinal disease, obtained 
Table 1 Abbreviations of analytes measured

\begin{tabular}{|c|c|}
\hline Full name & Abbreviation \\
\hline Chenodeoxycholic acid & CDCA \\
\hline Cholic acid & $\mathrm{CA}$ \\
\hline Deoxycholic acid & DCA \\
\hline Glycochenodeoxycholic acid & GCDCA \\
\hline Glycocholic acid & GCA \\
\hline Glycodeoxycholic acid & GDCA \\
\hline Glycoursodeoxycholic acid & GUDCA \\
\hline Lithocholic acid & LCA \\
\hline Taurochenodeoxycholic acid & TCDCA \\
\hline Taurocholic acid & TCA \\
\hline Taurodeoxycholic acid & TDCA \\
\hline Taurolithocholic acid & TLCA \\
\hline Tauroursodeoxycholic acid & TUDCA \\
\hline Ursodeoxycholic acid & UDCA \\
\hline 12-Oxolithocholic acid & 12-oxo-LCA \\
\hline 7-Oxodeoxycholic acid & 7-oxo-DCA \\
\hline 7-Oxohyocholic acid & 7-oxo-HCA \\
\hline$\beta$-Muricholic acid & $\beta \mathrm{MCA}$ \\
\hline $3 \alpha, 7 \alpha$-Dihydroxycholestanoic acid & DHCA \\
\hline Glycodehydrocholic acid & GDHCA \\
\hline Glycohyocholic acid & GHCA \\
\hline Glycohyodeoxycholic acid & GHDCA \\
\hline Glycolithocholic acid & GLCA \\
\hline Hyocholic acid & $\mathrm{HCA}$ \\
\hline Hyodeoxycholic acid & HDCA \\
\hline$\alpha, \beta$-Tauromuricholic acid & $\mathrm{T} \alpha \beta \mathrm{MCA}$ \\
\hline Taurohyodeoxycholic acid & TDHCA \\
\hline Taurodeoxycholic acid & THCA \\
\hline Taurohyodeoxycholic acid & THDCA \\
\hline$\omega$-Tauromuricholic acid & 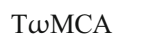 \\
\hline$\omega$-Tauromuricholic acid & $\omega \alpha \mathrm{MCA}$ \\
\hline Perfluorobutanoic acid & PFBA \\
\hline Perfluorobutane sulfonate & PFBS \\
\hline Perfluorodecanoic acid & PFDA \\
\hline Perfluorododecanoic acid & PFDoDA \\
\hline Perfluorododecane sulfonate & PFDoDS \\
\hline Perfluorodecane sulfonate & PFDS \\
\hline Potassium perfluoro-4-ethylcyclohexanesulfonate & PFECHS \\
\hline Perfluoroheptanoic acid & PFHpA \\
\hline Perfluoroheptane sulfonate & PFHpS \\
\hline Perfluorohexane sulfonate & PFHXS \\
\hline Perfluorononanoic acid & PFNA \\
\hline Perfluorononane sulfonate & PFNS \\
\hline Perfluorooctanoic acid & PFOA \\
\hline Linear-perfluorooctane sulfonate & L-PFOS \\
\hline Perfluorooctane sulfonamide & PFOSA \\
\hline Perfluoropentanoic acid & PFPeA \\
\hline Perfluoro pentane sulfonate & PFPeS \\
\hline Perfluorotetradecanoic acid & PFTDA \\
\hline Perfluorotridecanoic acid & PFTrDA \\
\hline Perfluoroundecanoic acid & PFUnDA \\
\hline
\end{tabular}

from Örebro University Hospital. The samples were collected and registered between years 2012 and 2014. All the participants gave written consent. All serum samples were stored at $-80{ }^{\circ} \mathrm{C}$ until analysis. Demographic characteristics of the study sample are shown in Electronic Supplementary Material (ESM) Table S1.

\section{Sample preparation}

The sample preparation procedure was performed as follows: all glassware and analytical syringes used were thoroughly rinsed with methanol (three times). Ten microliters of PFAS internal standard mixture $\left(c=200 \mathrm{ng} \mathrm{mL}^{-1}\right.$ in methanol; ESM Table S2) and $20 \mu \mathrm{L}$ of BA internal standard mixture $(c=$ $440-670 \mathrm{ng} \mathrm{mL}^{-1}$ in methanol; ESM Table S2) and $150 \mu \mathrm{L}$ serum or plasma were added to a 25-mg Ostro Protein Precipitation and Phospholipid Removal 96-well plate (Waters Corporation, Milford, USA), pre-conditioned with $450 \mu \mathrm{L}$ acetonitrile. A $450-\mu \mathrm{L}$ aliquot of acetonitrile (containing $1 \%$ formic acid) was added to all wells and mixed thoroughly with the sample by aspirating three times using an automated pipette. Samples were extracted using a 10" vacuum manifold for between 5 and $7 \mathrm{~min}$. Aliquots of $600 \mu \mathrm{L}$ of the eluate from each collection plate insert were then transferred to glass LC vials and evaporated to ca. $190 \mu \mathrm{L}$ using nitrogen. ${ }^{13} \mathrm{C}$-performance standards were added $(10 \mu \mathrm{L}$ of $200 \mathrm{ng} \mathrm{m} \mu \mathrm{L}^{-1}$ PFAS in methanol; ESM Table S2) as was $300 \mu \mathrm{L}$ of $2 \mathrm{mM} \mathrm{NH}_{4} \mathrm{AC}$ in water. All samples and standards were ultrasonicated for $10 \mathrm{~min}$ prior to instrumental analysis to ensure homogeneity. Samples that showed precipitation were centrifuged at $9900 \mathrm{~min}^{-1}$ for $10 \mathrm{~min}$. In addition, we tested the procedure with $20 \mu \mathrm{L}$ of serum, using the same internal standard mixtures and overall procedure, with two exceptions: (i) a frit filter plate was used which did not remove phospholipids (96-Well Protein Precipitation Filter Plate, Sigma-Aldrich) and (ii) after elution, the solvent was evaporated to dryness and the residue was dissolved in $20 \mu \mathrm{L}$ of a 40:60 MeOH to $\mathrm{H}_{2} \mathrm{O}$ v/v mixture containing the same ${ }^{13} \mathrm{C} /$ PFAS performance standards as the $150-\mu \mathrm{L}$ method.

\section{Method calibration curve}

Matrix-matched calibration standards were made using newborn bovine serum. In order to facilitate simultaneous analysis and subsequent quantification of PFASs and BAs, the newborn bovine serum was first filtered through an activated charcoal cartridge. Activated charcoal purification has previously been shown to efficiently remove planar molecules, including sterols, from biological samples [15] and was used to remove BAs present in high concentrations in the newborn bovine serum. Briefly, $3 \mathrm{~mL}$ of newborn bovine serum was filtered through an ENVI-Carb SPE cartridge $(250 \mathrm{mg}$ ) (SigmaAldrich, Steinheim, Germany) and used for subsequent calibration curves.

Matrix-matched calibration curves for PFASs The standards were prepared by spiking $150 \mu \mathrm{L}$ of pretreated newborn bovine serum with the native standard mixture resulting in an 8point matrix-matched curve ranging from 0.02 to $60 \mathrm{ng} \mathrm{mL}^{-1}$, 
including the matrix blank. The matrix-matched standards were further treated in the same way as authentic samples.

Matrix-matched calibration curves for BAs The standards were prepared by spiking $150 \mu \mathrm{L}$ of pretreated newborn bovine serum with the native standard mixture resulting in an 8point matrix-matched curve ranging from 1 to $320 \mathrm{ng} \mathrm{mL}^{-1}$, including the matrix blank. The matrix-matched standards were further treated in the same way as authentic samples.

\section{LC-MS analysis}

Analyses were performed on an Acquity UPLC system coupled to a triple quadrupole mass spectrometer (Waters Corporation, Milford, USA) with an atmospheric electrospray interface operating in negative ion mode. Aliquots of $10 \mu \mathrm{L}$ of samples were injected into the Acquity UPLC BEH C18 $2.1 \mathrm{~mm} \times$ $100 \mathrm{~mm}, 1.7-\mu \mathrm{m}$ column (Waters Corporation). A trap column (PFC Isolator column, Waters Corporation) was installed between the pump and injector and used to retain fluorinated compounds originating from the HPLC system and the mobile phase. The eluent system consisted of (A) $2 \mathrm{mM} \mathrm{NH}_{4} \mathrm{Ac}$ in water and (B) methanol (9:1) and $2 \mathrm{mM} \mathrm{NH}_{4} \mathrm{Ac}$ in methanol. The gradient was programmed as follows: $0-1 \mathrm{~min}, 1 \%$ solvent B; 1-13 min, $100 \%$ solvent B; $13-16$ min, $100 \%$ solvent B; 16-17 $\mathrm{min}, 1 \%$ solvent $\mathrm{B}$, flow rate $0.3 \mathrm{~mL} / \mathrm{min}$. The total run time for UPLC-MS/MS analysis was $17 \mathrm{~min}$, while the total run time for each sample injection was $20 \mathrm{~min}$, including the reconditioning of the analytical column.

MS analysis was performed in multiple reaction monitoring (MRM) mode and experimental details of the MS/MS method are given in ESM Table S2. The cone and collision energies were optimized for each analyte along with the precursor ion and product ion $(\mathrm{m} / \mathrm{z})$ which is shown with the abbreviations in ESM Table S2. Monitoring of the transitions between molecular anion $[\mathrm{M}-\mathrm{H}]^{-}$for the PFCAs and PFSAs and one product ion; [M$\mathrm{COOH}]^{-}$and $\left[\mathrm{FSO}_{3}\right]^{-}$were used for quantification of PFCAs and PFSAs, respectively. Additional 1-2 product ions were monitored as qualification ions except for PFPeA and PFHxA, for which only one product ion was monitored. For BAs, monitoring of the transitions between molecular anion $[\mathrm{M}-\mathrm{H}]^{-}$and one to two product ions including $\left[\mathrm{SO}_{3}\right]^{-}$, [taurine- $\left.\mathrm{H}\right]^{-},\left[\mathrm{CH}_{2} \mathrm{CHSO}_{3}\right]^{-}$, and $\left[\mathrm{NH}_{2} \mathrm{CH}_{2} \mathrm{COO}\right]^{-}$for the conjugated BAs and $\left.[\mathrm{M}-\mathrm{H}]\right]^{-}$and $[\mathrm{M}-\mathrm{H}-$ $\left.2 \mathrm{H}_{2} \mathrm{O}\right]^{-}$for the non-conjugated BAs.

\section{Method validation}

For method validation, the following parameters were investigated: linearity, method detection limit (MDL), repeatability, accuracy and precision, recovery, and matrix effect. Linearity was determined with matrix-matched standards, and plotted as relative peak areas (analyte/internal standard) versus analyte concentration.

\section{Results and discussion}

The analytical method was a combination of two methods that we developed earlier for BAs and PFASs $[12,16]$. The main aim was to simultaneously determine both compound classes to enable human PFAS exposure studies to investigate a potential interaction of serum/plasma PFAS and BA concentrations (Fig. 1). The method was validated for both $150 \mu \mathrm{L}$ and $20 \mu \mathrm{L}$ of serum/plasma.

\section{Validation}

Specificity The method provided good chromatographic robustness, with the deviation of retention times within a time margin of $\pm 2.5 \%$. For the majority of compounds, multiple product ion fragments were monitored. In the case of the known co-eluting compounds, at least two product ions from the specific precursor ion were monitored with the requirement that the ion ratio for the secondary product ion be within $50 \%$ variation from the selected quantification reference. For example, in the case of LPFOS and TCDCA and TDCA (including TUDCA and THDCA) (which share the same mass of product ion $\mathrm{m} / \mathrm{z}=$ 499 and major fragment at $m / z=80$ and tend to co-elute), we specifically monitored two different (other than $499>80$ ) product ions unique to the two compounds in order to ensure interference-free quantitation (ESM Fig. S1).

Linearity The linearity $\left(R^{2}\right)$ of the matrix-matched calibration curves ranged from 0.9995 to 0.99998 for PFASs and from 0.9906 to 0.9997 for BAs. The relative response factors (RRFs) of the calibration curve ranged between 0.603 and 1.47 for PFASs and between 0.23 and 1.70 for BAs with relative standard deviation $<15 \%$ for all major serum PFASs and $<$ $22 \%$ for the broad range of BAs with the exception of three BAs above $25 \%$ : $\mathrm{CA}(26 \%)$, $\mathrm{HCA}(27 \%)$, and $\omega \alpha \mathrm{MCA}(31 \%)$.

Sensitivity MDLs were determined by calculating the mean concentrations plus three times the standard deviation in water blanks run with the sample batch $(n=7)$. Overall, the method performed well, giving MDLs in the range of $0.01-0.06 \mathrm{ng}$ $\mathrm{mL}^{-1}$ for PFAS using $150 \mu \mathrm{L}$ of sample. The MDLs for BAs were in the range of $0.002-0.152 \mathrm{ng} \mathrm{mL}^{-1}$, with the exception of CA $\left(0.1 \mathrm{ng} \mathrm{mL} L^{-1}\right)$ and GCDCA $\left(0.56 \mathrm{ng} \mathrm{mL}^{-1}\right)$, which gave higher MDLs due to higher background noise in the water blanks. With a $20-\mu \mathrm{L}$ sample volume, the MLDs were in the range of $0.02-0.5 \mathrm{ng} \mathrm{mL}^{-1}$ for all PFASs except for PFUnDA, for which the MDL was $10 \mathrm{ng} \mathrm{mL}^{-1}$. For BAs, the MLDs were in the range of 0.0025 to $1.0 \mathrm{ng} \mathrm{mL}^{-1}$ with the exception of wMCA and 12-oxo-LCA which had higher $\operatorname{MDL}\left(10 \mathrm{ng} \mathrm{mL}^{-1}\right)$.

Accuracy and precision The method provided accurate data and conformed well to certified serum PFAS concentrations 


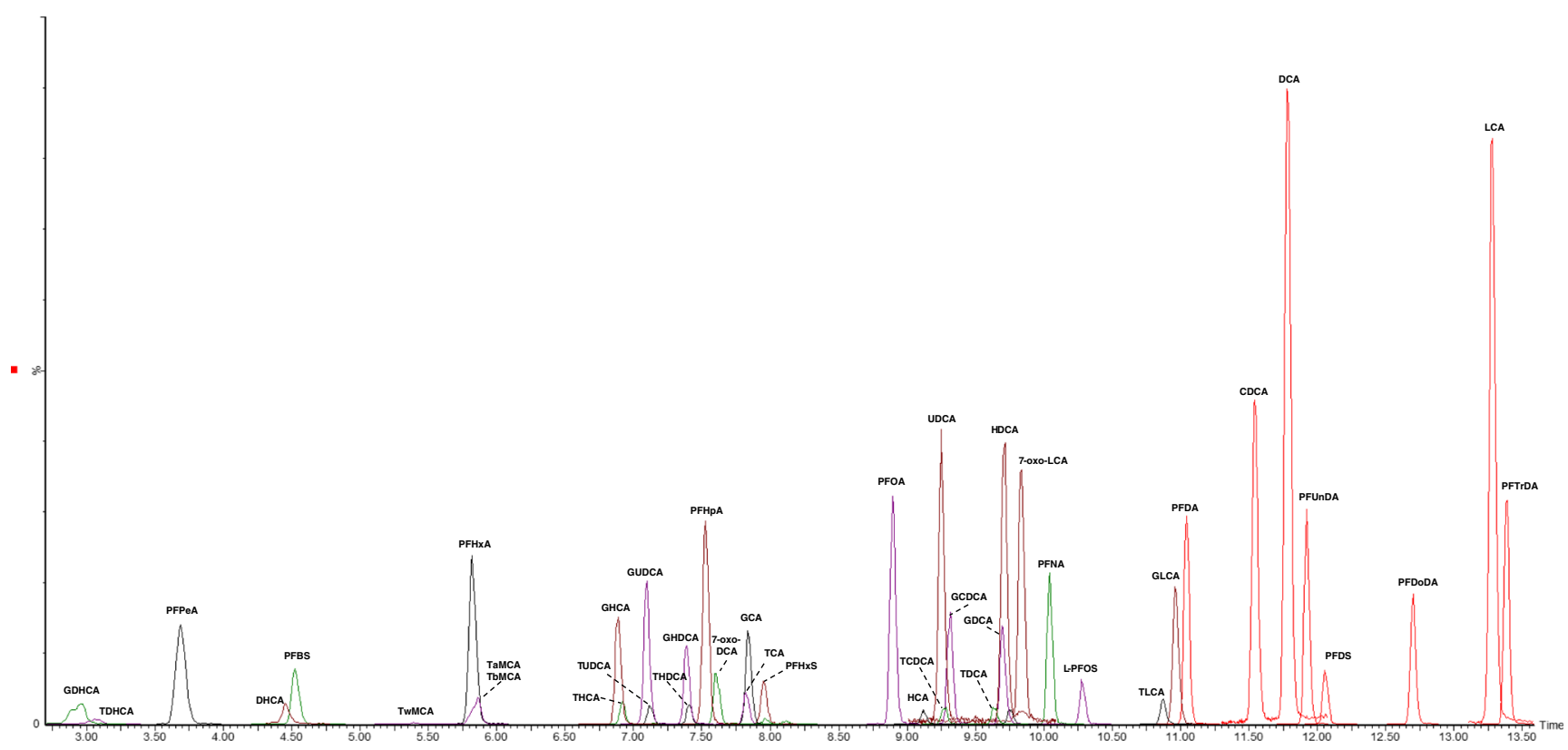

Fig. 1 Example extracted ion chromatogram of target analytes in elution order, in accordance with ESM Table S2

of NIST SRM 1957. The method was reproducible with relative standard deviations ( $n=10$, NIST and in-house QC plasma), ranged from 2 to $7 \%$ for all major plasma PFAS, with the exception of PFUnDA at $24 \%$ in the NIST sample (Fig. 2, ESM Table S3). The compounds had a lower recovery, most likely due to matrix effects in that sample, as the recovery and repeatability were clearly better in the in-house QC sample. We are unaware of any NIST SRM-certified values for BAs and thus performed plasma spike tests at four five levels, 0, 50, 100, 200, and $400 \mathrm{ng} / \mathrm{mL}$ of 35 BAs. The matrix spike test results demonstrated our ability to detect BAs within an error range of $20 \%$, with recoveries ranging from 79 to $106 \%$ for most BAs (ESM Table S4). We also evaluated potential matrix ionization enhancement or suppression using nine internal BA standards and the PFAS performance standards which were added to extract prior to injection both to determine the internal standard recovery and to monitor the trending instrument performance at two concentration levels, 50 and $100 \mathrm{ng} / \mathrm{mL}$ (ESM Fig. S2). We did not observe any deviations, other than those expected at $25 \%$ difference. Similar differences were observed for PFASs. Taken together, we can conclude that the method enables simultaneous analysis of PFASs and BAs. We also analyzed ten pooled serum samples using 20 $\mu \mathrm{L}$ of sample, and the average RSDs for both the PFAS and BA were $<10 \%$ (ESM Table S5). However, some of the PFASs and BAs were below the MLD of the method. Overall, it is possible to reduce the sample volume, however, with some compromises in the sensitivity of the method.

Comparing the here-presented combined PFAS and BA method to the previously reported separate methods for these
Fig. 2 Conformity of measured PFAS concentration with certified values in NIST SRM 1957. 95\% confidence intervals $(\mathrm{CI})$ are (i) based on 4 replicates (our method) or (ii) based on published CI (NIST SRM 1957) [32]

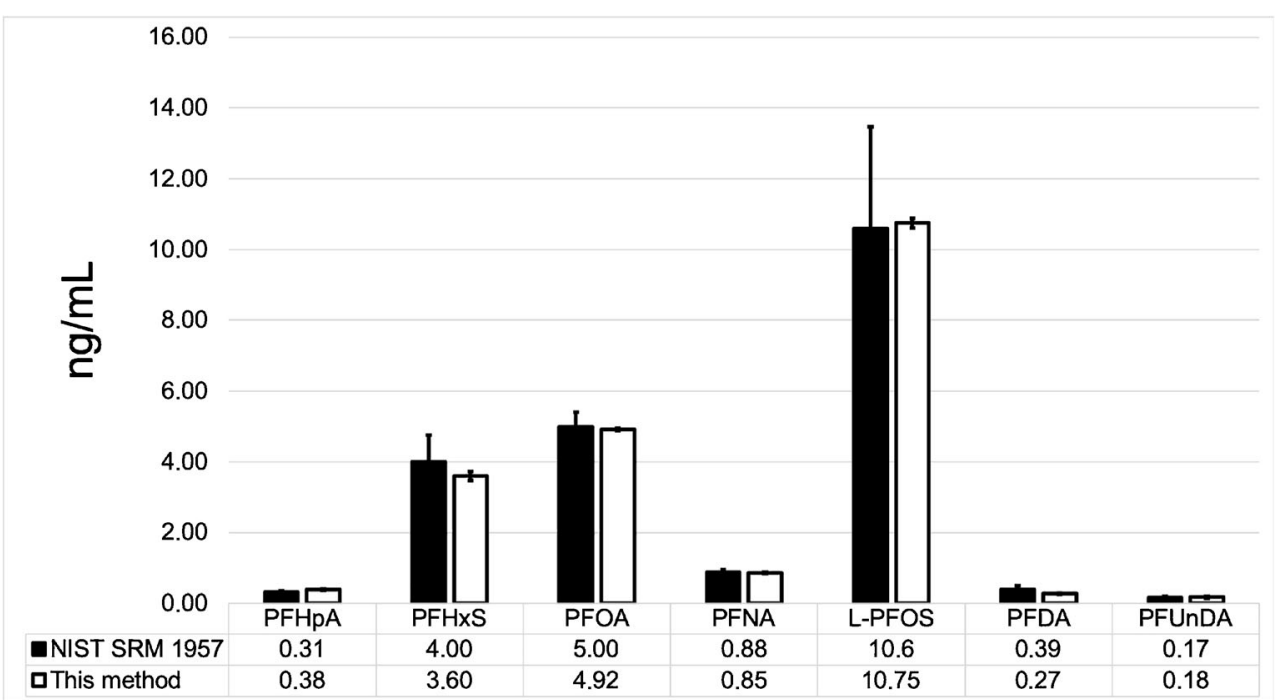


Table 2 Measured concentration values from 20 healthy individuals Median concentration Min. $(\mathrm{ng} / \mathrm{mL}) \quad$ Max. $(\mathrm{ng} / \mathrm{mL})$ (ng/mL)

\begin{tabular}{|c|c|c|c|}
\hline $\mathrm{CA}$ & 30.21 & 5.14 & 537.29 \\
\hline CDCA & 80.11 & 9.87 & 606.25 \\
\hline GCA & 254.40 & 64.07 & 1039.72 \\
\hline GCDCA & 761.93 & 90.22 & 2113.96 \\
\hline TCDCA & 97.92 & 11.39 & 371.25 \\
\hline 12-oxo-LCA & 13.26 & 2.45 & 34.82 \\
\hline DCA & 240.82 & 0.01 & 737.10 \\
\hline HDCA & 86.99 & 8.48 & 498.08 \\
\hline LCA & 6.73 & 0.00 & 21.96 \\
\hline UDCA & 30.12 & 16.76 & 194.31 \\
\hline GDCA & 295.31 & 0.14 & 2154.80 \\
\hline GHCA & 5.66 & 2.50 & 15.89 \\
\hline GHDCA & 7.60 & 0.05 & 46.41 \\
\hline GLCA & 20.32 & 4.25 & 141.00 \\
\hline GUDCA & 34.94 & 4.49 & 264.67 \\
\hline $\mathrm{T} \alpha \beta \mathrm{MCA}$ & 1.97 & 0.00 & 17.94 \\
\hline TDCA & 35.69 & 2.37 & 113.93 \\
\hline THCA & 1.60 & 0.00 & 7.71 \\
\hline TLCA & 3.19 & 1.09 & 12.60 \\
\hline PFHxS & 0.78 & 0.07 & 6.18 \\
\hline PFOA & 1.42 & 0.15 & 3.48 \\
\hline PFNA & 0.76 & 0.06 & 2.03 \\
\hline L-PFOS & 4.20 & 0.44 & 16.69 \\
\hline PFDA & 0.37 & 0.04 & 0.84 \\
\hline PFUnDA & 0.39 & 0.06 & 0.91 \\
\hline PFTrDA & 0.08 & 0.01 & 0.20 \\
\hline
\end{tabular}

two groups of compounds, the novel method allowed us to minimize the sample consumption, while allowing faster analysis with similar sensitivity, linear range, and accuracy. Since it was possible to reduce the sample volume down to $20 \mu \mathrm{L}$, without substantial loss of sensitivity or accuracy, our approach is a viable option when the sample volume is limited. However, if the goal is to perform a comprehensive determination of BA and PFAS profiles, the use of larger sample volume is recommended. Methods developed for PFASs typically report LOD/LOQ values ranging from 0.018 to $0.15 \mathrm{ng} /$ ML [13, 16, 14], and for BAs in the range of 0.1 to $50 \mathrm{ng} / \mathrm{mL}$ $[12,17,18]$, which are very similar to our values obtained, when using the $150-\mu \mathrm{L}$ extraction. As demonstrated with the analysis of the certified NIST serum sample, our reported method was highly accurate for PFAS. For BAs, no certified material was available; however, the spiked samples gave robust results. Here, we have used matrix-matched calibration for PFAS and BA after matrix purification. Application of the matrix-matched calibration for PFAS analyses has improved precision; however, since the internal standards are applied in the quantitation, the impact of the possible matrix suppression would not significantly hamper the accuracy of the method substantially, as has been shown also in inter-laboratory studies [19]. In our study, based on the evaluation of the matrix effects (ESM Fig. S2), the effect was in average $<20 \%$. Thus,
Fig. 3 Correlation plot of PFASs and BAs (Spearman's correlation), significance of the correlations is marked $(* * * p<$ $0.01 ; * p<0.05 ; * p<0.1)$

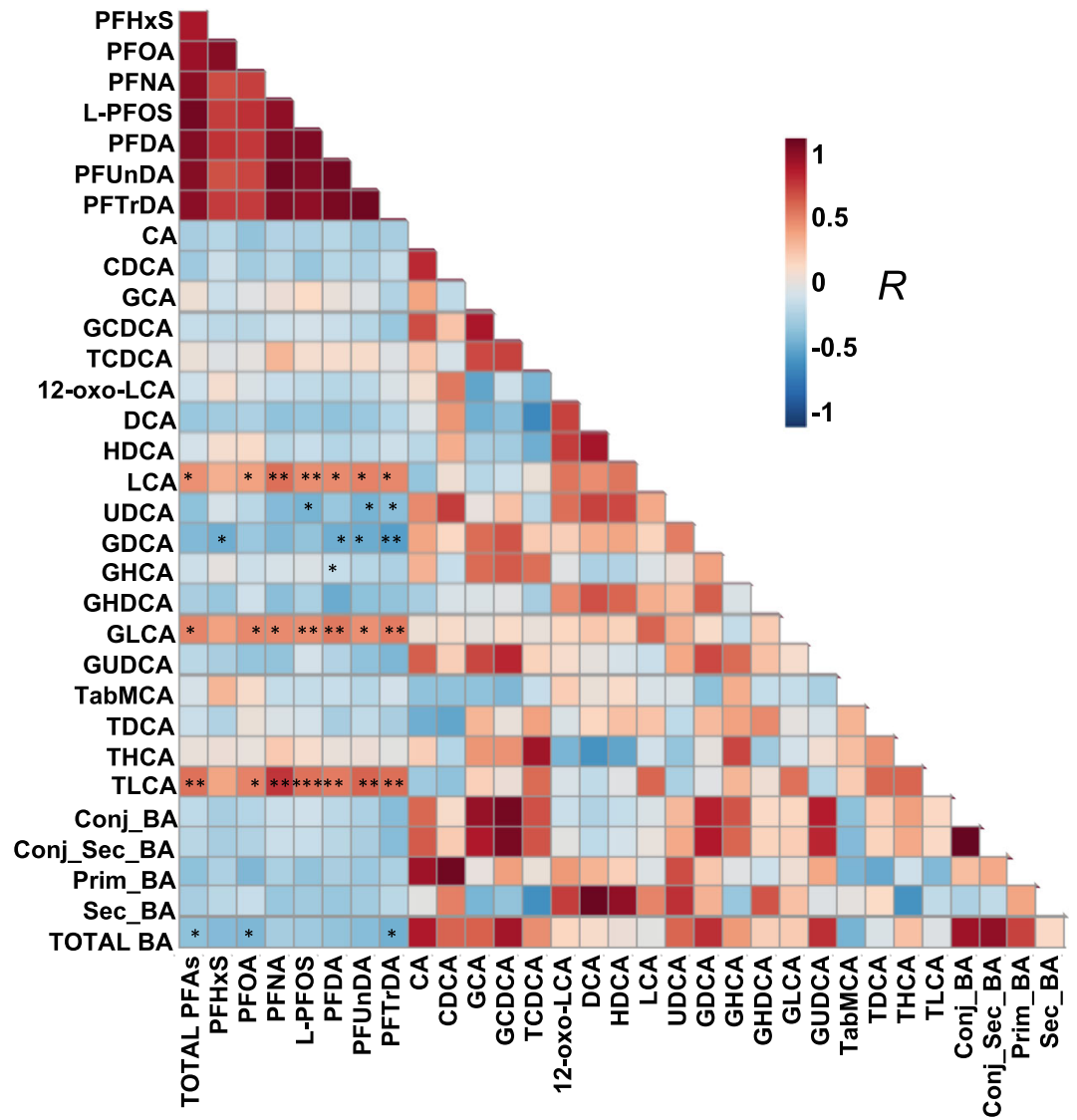


application of a single calibration mixture of pure standards is, in principle, a feasible option, without a major impact on the precision of quantitation.

\section{PFASs and BAs in human serum}

The median concentrations of the PFASs and BAs in a series of 20 samples from healthy human individuals are shown in Table 2. Of the 20 measured PFASs, seven PFASs could be detected in a majority of the samples, and 19 bile acids were detected in > $70 \%$ of the samples, and these were taken for further data analysis. In the measured set of samples, the age or the sex did not have a significant impact on the measured concentrations.

Next, we studied the correlation between PFAS and BA concentrations. As can be seen in Fig. 3, specific bile acids, namely LCA, GDCA, GLCA, and TLCA, showed significant associations with PFAS concentrations, with negative association between GDCA and positive associations between lithocholic acids and its two conjugates (GLCA and TLCA). The overall trend, although not reaching statistically significant in all compounds, was that the majority of circulating BAs were negatively associated with PFAS. This would suggest that the de novo synthesis of BAs is downregulated, in accordance with the literature. Specifically, our findings are in line with a previous report, where several PFASs were found to suppress CYP7A1, an enzyme that controls the first and rate-limiting step in the formation of BAs from cholesterol [11].

The increased levels of LCA and its two conjugated BAs, on the other hand, could indicate any of (i) increased re-uptake of the bile acids in the gut, (ii) decreased clearance from the blood, (iii) increased production of the conjugated bile acids in the liver, or (iv) decreased de-conjugation of them by the microbiota, or any combination of these. Additionally, our results appear to suggest increased microbial formation of LCA. Indeed, PFAS exposure has been shown to cause alteration in gut microbiota, with higher exposure to PFAS associated with reduced microbiome diversity [20]. On the other hand, the decreased fecal BA excretion, linked with PFAS exposure [11], may be nonexclusively due to inhibition of the biosynthesis of the BAs $[8,21,10]$ or due to the increased uptake of BAs in the gut. It has been shown that PFOA inhibits the function of the hepatocyte nuclear factor $4 \alpha$ [22], which plays a central role in the regulation of BA metabolism in the liver, and is linked both with the synthesis and conjugation of primary BAs. Overall,

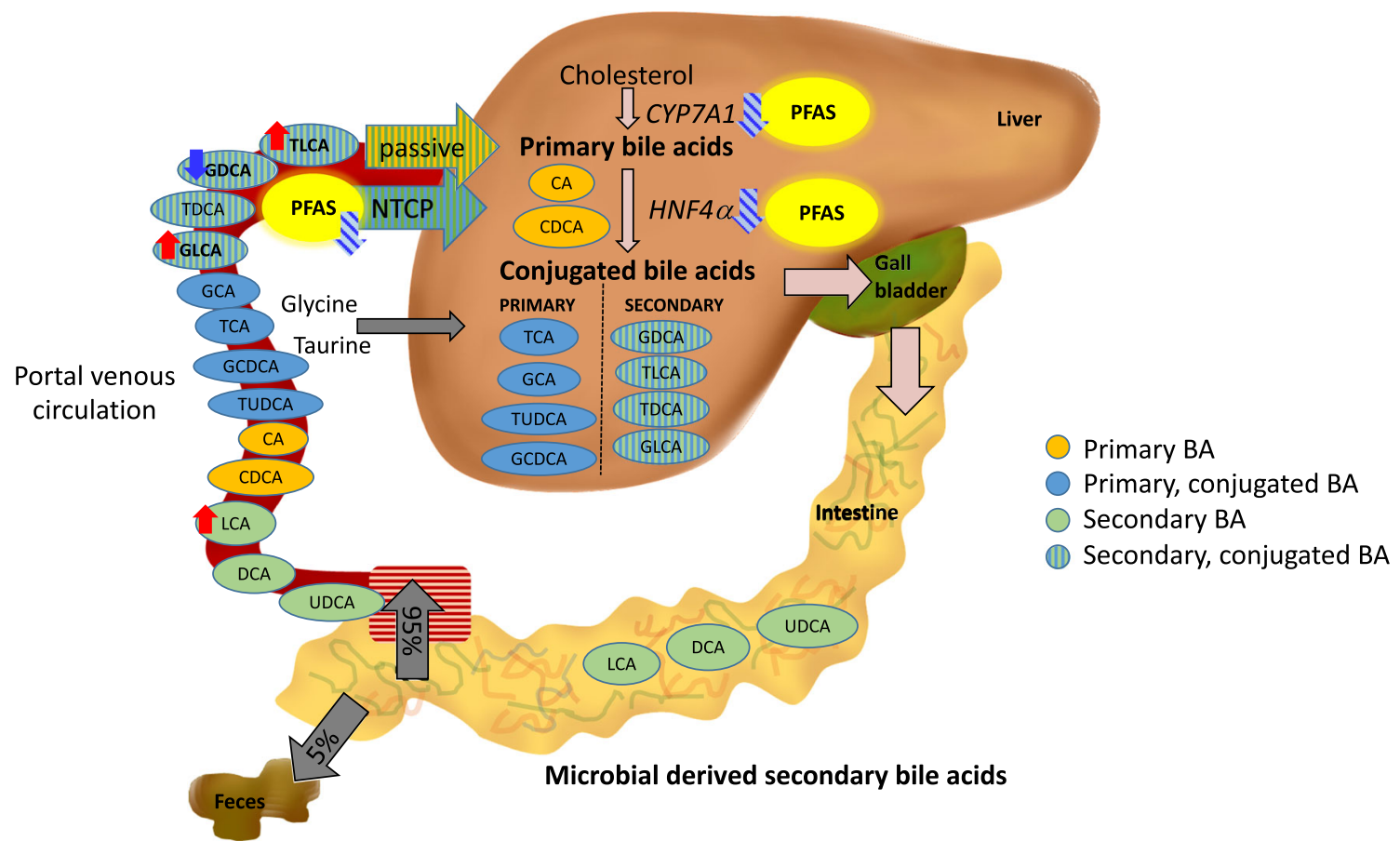

Fig. 4 The enterohepatic circulation of bile acids. Primary bile acids (CA, cholic acid; CDCA, chenodeoxycholic acid) are synthetized from cholesterol in the liver, with the first step controlled primarily via the action of cholesterol $7 \alpha$-hydroxylase (CYP7A1) which is downregulated by PFAS. Before the primary bile acids are secreted into the canalicular lumen, they are conjugated with either of the amino acids, glycine or taurine. HNF $4 \alpha$ can regulate the genes involved in BA biosynthesis, including hydroxylation and side chain $\beta$-oxidation of cholesterol in vivo. Once in the large intestine, bacterial flora catalyzes their biotransformation into secondary bile acids: deoxycholic acid (DCA) and lithocholic acid (LCA). Ursodeoxycholic acid (UDCA) derives from epimerization of CDCA. From the colon, around 95\% are reabsorbed into the distal ileum. The absorbed primary and secondary bile acids and salts are transported back to the liver where most of the conjugated BAs as well as PFASs are actively transported into hepatocytes by sodium $(\mathrm{Na}+$ taurocholate co-transporting polypeptide (NTCP). Once in the liver, the BAs are reconjugated and then re-secreted together with newly synthesized bile salts. Red arrows, positive association with PFAS; blue arrow, negative association with PFAS. The impacts on CYP7A1, HNF4a, and NTCP are based on the literature $[10,8,21,22]$. 
there was a negative association between conjugated BAs and PFASs, and thus, it is more likely that the observed increase of the two conjugated BAs is related to either their re-uptake or decreased de-conjugation (Fig. 4). The liver clears most of the BAs via sodium taurocholate co-transporting polypeptide (NTCP), which has a high affinity for all conjugated BAs, while the unconjugated BAs, such as LCA, are using mainly passive transport [23]. Interestingly, it has been demonstrated that PFBS, PFHxS, and PFOS are also substrates for human NTCP [8]. However, as LCA is transported passively, the changes observed are more likely due to the increased uptake from the gut than due to increased clearance. Interestingly, LCA, but not the other BAs, is an activator of nuclear receptor vitamin D receptor (VDR), which in turn regulates the intestinal barrier functions. It has been suggested that activation of VDR may be involved in increasing BA absorption and in suppressing hepatic BA synthesis [24-26].

Taken together, our observed associations between PFASs and BAs are potentially important for our understanding of cardiometabolic diseases, which is because BA metabolism is known to play a role in the pathogenesis of type 2 diabetes (T2D), atherosclerosis, and non-alcoholic fatty liver disease (NAFLD) [27]. Taurine-conjugated BAs have, for example, been found to be elevated in T2D [28]. Moreover, LCA can be cytotoxic, leading to oxidative stress, membrane damage, and colonic carcinogenesis [29], while TLCA is known to induce cholestasis by impairing biliary BA secretion $[30,31]$.

\section{Conclusions}

The method presented here is suitable for fast, automated analysis of PFASs and BAs from human serum, and the sample amount can be reduced to $20 \mu \mathrm{L}$, however, with some loss of sensitivity. Our validation of the method demonstrated that the method is robust and accurate. Our preliminary findings that various PFASs have significant association with BAs support the notion that they may play a role in the health impacts of PFAS exposure, such as the known impact of PFAS on cholesterol levels, and in metabolic pathologies such as T2D and NAFLD. Taken together, our findings warrant further investigation of the impact of both specific and mixtures of PFAS on BA metabolism, including the potential role of gut microbiota. Such studies may provide valuable insight into the pathogenesis and varying incidence of common metabolic and immune-mediated inflammatory disorders.

Acknowledgments Open access funding provided by Örebro University. The authors thank Dr. Annie Von Eyken Bonafonte (University of Turku) for technical assistance and Dr. Aidan McGlinchey (Örebro University) for editing.

Funding information This study was financially supported by funding from Vetenskapsrådet (to TH; grant no. 2016-05176).

\section{Compliance with ethical standards}

The serum samples from healthy human subjects were collected at Örebro University Hospital. All participants have given written informed consent prior to participation. The study was performed according to the Declaration of Helsinki and was approved by the Uppsala regional Ethical Committee (Dnr. 2006/245).

Conflict of interest Author JH reported receiving consulting and lecture fees from AbbVie, Celgene, Celltrion, Ferring, Hospira, Janssen, Medivir, MSD, Pfizer, Prometheus Laboratories Inc., Sandoz, Shire, Takeda, Thermo Fisher Scientific, Tillotts Pharma, and Vifor Pharma, and research grants from Janssen, MSD, and Takeda. Author IS reported receiving lecture fees from Nutricia and Meda. Authors SS, AMD, FF, LS, TL, $\mathrm{MO}$, and TH declare that they have no conflict of interest.

Open Access This article is distributed under the terms of the Creative Commons Attribution 4.0 International License (http:// creativecommons.org/licenses/by/4.0/), which permits unrestricted use, distribution, and reproduction in any medium, provided you give appropriate credit to the original author(s) and the source, provide a link to the Creative Commons license, and indicate if changes were made.

\section{References}

1. Haeusler RA, Astiarraga B, Camastra S, Accili D, Ferrannini E. Human insulin resistance is associated with increased plasma levels of 12a-hydroxylated bile acids. Diabetes. 2013;62(12):4184-91. https://doi.org/10.2337/db13-0639.

2. Prawitt J, Caron S, Staels B. Bile acid metabolism and the pathogenesis of type 2 diabetes. Curr Diab Rep. 2011;11(3):160. https:// doi.org/10.1007/s11892-011-0187-x.

3. Kingsley SL, Walker DI, Calafat AM, Chen A, Papandonatos GD, $\mathrm{Xu}$ Y, et al. Metabolomics of childhood exposure to perfluoroalkyl substances: a cross-sectional study. Metabolomics. 2019;15(7):95. https://doi.org/10.1007/s11306-019-1560-z.

4. Land M, de Wit CA, Bignert A, Cousins IT, Herzke D, Johansson $\mathrm{JH}$, et al. What is the effect of phasing out long-chain per- and polyfluoroalkyl substances on the concentrations of perfluoroalkyl acids and their precursors in the environment? A systematic review. Environ Evid. 2018;7(1):4. https://doi.org/10.1186/s13750-0170114-y.

5. Averina M, Brox J, Huber S, Furberg AS. Perfluoroalkyl substances in adolescents in northern Norway: lifestyle and dietary predictors. The Tromso study, Fit Futures 1. Environ Int. 2018;114:123-30. https://doi.org/10.1016/j.envint.2018.02.031.

6. Sun Q, Zong G, Valvi D, Nielsen F, Coull B, Grandjean P. Plasma concentrations of perfluoroalkyl substances and risk of type 2 diabetes: a prospective investigation among U.S. women. Environ Health Perspect. 2018;126(3):037001. https://doi.org/10.1289/ EHP2619.

7. Winkens K, Vestergren R, Berger U, Cousins IT. Early life exposure to per- and polyfluoroalkyl substances (PFASs): a critical review. Emerg Contam. 2017;3(2):55-68. https://doi.org/10.1016/j.emcon. 2017.05.001.

8. Zhao W, Zitzow JD, Ehresman DJ, Chang S-C, Butenhoff JL, Forster J, et al. Na+/taurocholate cotransporting polypeptide and apical sodium-dependent bile acid transporter are involved in the disposition of perfluoroalkyl sulfonates in humans and rats. Toxicol Sci. 2015;146(2):363-73. https://doi.org/10.1093/toxsci/kfv102. 
9. Chiang JY. Recent advances in understanding bile acid homeostasis. F1000Res. 2017;6:2029. https://doi.org/10.12688/ f1000research.12449.1.

10. Beggs KM, McGreal SR, McCarthy A, Gunewardena S, Lampe $\mathrm{JN}$, Lau C, et al. The role of hepatocyte nuclear factor 4-alpha in perfluorooctanoic acid- and perfluorooctanesulfonic acid-induced hepatocellular dysfunction. Toxicol Appl Pharmacol. 2016;304: 18-29. https://doi.org/10.1016/j.taap.2016.05.001.

11. Bijland S, Rensen PC, Pieterman EJ, Maas AC, van der Hoorn JW, van Erk MJ, et al. Perfluoroalkyl sulfonates cause alkyl chain length-dependent hepatic steatosis and hypolipidemia mainly by impairing lipoprotein production in APOE*3-Leiden CETP mice. Toxicol Sci. 2011;123(1):290-303. https://doi.org/10.1093/toxsci/ kfr142.

12. Jantti SE, Kivilompolo M, Ohrnberg L, Pietilainen KH, Nygren H, Oresic M, et al. Quantitative profiling of bile acids in blood, adipose tissue, intestine, and gall bladder samples using ultra high performance liquid chromatography-tandem mass spectrometry. Anal Bioanal Chem. 2014;406(30):7799-815. https://doi.org/10.1007/ s00216-014-8230-9.

13. Gao K, Fu J, Xue Q, Li Y, Liang Y, Pan Y, et al. An integrated method for simultaneously determining 10 classes of per- and polyfluoroalkyl substances in one drop of human serum. Anal Chim Acta. 2018;999:76-86. https://doi.org/10.1016/j.aca.2017. 10.038 .

14. Yu CH, Patel B, Palencia M, Fan Z. A sensitive and accurate method for the determination of perfluoroalkyl and polyfluoroalkyl substances in human serum using a high performance liquid chromatography-online solid phase extraction-tandem mass spectrometry. J Chromatogr A. 2017;1480:1-10. https://doi.org/10. 1016/j.chroma.2016.11.063

15. Lacina O, Hradkova P, Pulkrabova J, Hajslova J. Simple, high throughput ultra-high performance liquid chromatography/tandem mass spectrometry trace analysis of perfluorinated alkylated substances in food of animal origin: milk and fish. J Chromatogr A. 2011;1218(28):4312-21. https://doi.org/10.1016/j.chroma.2011. 04.061 .

16. Salihovic S, Kärrman A, Lindström G, Lind PM, Lind L, van Bavel B. A rapid method for the determination of perfluoroalkyl substances including structural isomers of perfluorooctane sulfonic acid in human serum using 96-well plates and column-switching ultra-high performance liquid chromatography tandem mass spectrometry. J Chromatogr A. 2013;1305:164-70. https://doi.org/10. 1016/j.chroma.2013.07.026.

17. Han J, Liu Y, Wang R, Yang J, Ling V, Borchers CH. Metabolic profiling of bile acids in human and mouse blood by LC-MS/MS in combination with phospholipid-depletion solid-phase extraction. Anal Chem. 2015;87(2):1127-36. https://doi.org/10.1021/ ac503816u.

18. Ghaffarzadegan T, Essén S, Verbrugghe P, Marungruang N, Hållenius FF, Nyman M, et al. Determination of free and conjugated bile acids in serum of Apoe(-/-) mice fed different lingonberry fractions by UHPLC-MS. Sci Rep. 2019;9(1):3800. https://doi.org/ 10.1038/s41598-019-40272-8.

19. Longnecker MP, Smith CS, Kissling GE, Hoppin JA, Butenhoff JL, Decker E, et al. An interlaboratory study of perfluorinated alkyl compound levels in human plasma. Environ Res. 2008;107(2): 152-9. https://doi.org/10.1016/j.envres.2008.01.005.
20. Iszatt N, Janssen S, Lenters V, Dahl C, Stigum H, Knight R, et al. Environmental toxicants in breast milk of Norwegian mothers and gut bacteria composition and metabolites in their infants at 1 month. Microbiome. 2019;7(1):34. https://doi.org/10.1186/s40168-0190645-2.

21. Zhao W, Zitzow JD, Weaver Y, Ehresman DJ, Chang S-C, Butenhoff JL, et al. Organic anion transporting polypeptides contribute to the disposition of perfluoroalkyl acids in humans and rats. Toxicol Sci. 2017;156(1):84-95. https://doi.org/10.1093/toxsci/ kfw236.

22. Buhrke T, Kruger E, Pevny S, Rossler M, Bitter K, Lampen A. Perfluorooctanoic acid (PFOA) affects distinct molecular signalling pathways in human primary hepatocytes. Toxicology. 2015;333: 53-62. https://doi.org/10.1016/j.tox.2015.04.004.

23. Dawson PA. Role of the intestinal bile acid transporters in bile acid and drug disposition. Handb Exp Pharmacol. 2011;201:169-203. https://doi.org/10.1007/978-3-642-14541-4_4.

24. Bakke D, Chatterjee I, Agrawal A, Dai Y, Sun J. Regulation of microbiota by vitamin D receptor: a nuclear weapon in metabolic diseases. Nucl Receptor Res. 2018;5:101377. https://doi.org/10. 11131/2018/101377.

25. Ticho AL, Malhotra P, Dudeja PK, Gill RK, Alrefai WA. Bile acid receptors and gastrointestinal functions. Liver Research. 2019;3(1): 31-9. https://doi.org/10.1016/j.livres.2019.01.001.

26. Han S, Li T, Ellis E, Strom S, Chiang JYL. A novel bile acidactivated vitamin $\mathrm{D}$ receptor signaling in human hepatocytes. Mol Endocrinol. 2010;24(6):1151-64. https://doi.org/10.1210/me. 2009-0482.

27. de Aguiar Vallim Thomas Q, Tarling Elizabeth J, Edwards Peter A. Pleiotropic roles of bile acids in metabolism. Cell Metabolism. 2013;17(5):657-69. https://doi.org/10.1016/j.cmet.2013.03.013.

28. Wewalka M, Patti ME, Barbato C, Houten SM, Goldfine AB. Fasting serum taurine-conjugated bile acids are elevated in type 2 diabetes and do not change with intensification of insulin. J Clin Endocrinol Metab. 2014;99(4):1442-51. https://doi.org/10.1210/jc. 2013-3367.

29. Barrasa JI, Olmo N, Lizarbe MA, Turnay J. Bile acids in the colon, from healthy to cytotoxic molecules. Toxicol in Vitro. 2013;27(2): 964-77. https://doi.org/10.1016/j.tiv.2012.12.020.

30. Kakis GYI. Pathogenesis of lithocholate- and taurolithocholateinduced intrahepatic cholestasis in rats. Gastroenterology. 1978;4: 13.

31. Crocenzi FA, Mottino AD, Sánchez Pozzi EJ, Pellegrino JM, Rodríguez Garay EA, Milkiewicz P, et al. Impaired localisation and transport function of canalicular Bsep in taurolithocholate induced cholestasis in the rat. Gut. 2003;52(8):1170-7. https://doi. org/10.1136/gut.52.8.1170.

32. Schantz MM, Eppe G, Focant JF, Hamilton C, Heckert NA, Heltsley RM, et al. Milk and serum standard reference materials for monitoring organic contaminants in human samples. Anal Bioanal Chem. 2013;405(4):1203-11. https://doi.org/10.1007/ s00216-012-6524-3.

Publisher's note Springer Nature remains neutral with regard to jurisdictional claims in published maps and institutional affiliations. 